\title{
Probiotic Supplementation Reduces Inflammatory Profiles But Does Not Prevent Oral Immune Perturbations During SIV Infection
}

Rhianna Jones ${ }^{1}$, Kyle Kroll ${ }^{1}$, Courtney Broedlow ${ }^{2,3}$, Luca Schifanella², Scott Smith ${ }^{1}$, Brady Hueber ${ }^{1}$, Spandan V. Shah, Daniel R. Ram, Cordelia Manickam, Valerie Varner ${ }^{1}$, Nichole R. Klatt ${ }^{2,3}$, R. Keith Reeves ${ }^{1,4^{*}}$

${ }^{1}$ Center for Virology and Vaccine Research, Beth Israel Deaconess Medical Center, Harvard Medical School, Boston, MA; ${ }^{2}$ Division of Surgical Outcomes and Precision Medicine Research, Department of Surgery, University of Minnesota, Minneapolis, MN, USA; ${ }^{3}$ Department of Pharmaceutics, University of Washington, Seattle, WA, USA;

${ }^{4}$ Ragon Institute of Massachusetts General Hospital, MIT, and Harvard, Cambridge, MA 02139, USA

${ }^{*}$ Corresponding author

R. Keith Reeves

Center for Virology and Vaccine Research

Beth Israel Deaconess Medical Center

3 Blackfan Circle

Boston, MA 02215

Ph: (617-735-4586)

Fax: (617-735-4527)

E-mail: rreeves@bidmc.harvard.edu

Abstract: 200 words

Text: 3209 words

References: 50

Figures: 7

Supplementary Figures: 1

Supplementary Tables: 1

Running title: Probiotics and SIV Infection

Funding acknowledgement: This work was supported by R01 DE026327, R01 DE026014, and R21 Al145678 (to R.K.R.). The authors also acknowledge the CFAR/CVVR Flow Cytometry Core (P30 Al060354). 


\section{ABSTRACT}

49 HIVISIV infections lead to massive loss of mucosal CD4+ T cells and breakdown of the

50 epithelial mucosa resulting in severe microbial dysbiosis and chronic immune activation

51 that ultimately drive disease progression. Moreover, disruption of one of the most

52 understudied mucosal environments, the oral cavity, during HIV-induced

53 immunosuppression results in significant microbial and neoplastic co-morbidities and

54 contributes to and predicts distal disease complications. In this study we evaluated the

55 effects of oral probiotic supplementation $(\mathrm{Pbx})$, which can stimulate and augment

56 inflammatory or anti-inflammatory pathways, on early SIV infection of rhesus macaques.

57 Our study revealed that similar to the GI mucosae, oral CD4+ T cells were rapidly

58 depleted, and as one of the first comprehensive analyses of the oral microflora in SIV

59 infection, we also observed significant modulation among two genera, Porphyromonas

60 and Actinobacillus, early after infection. Interestingly, although $\mathrm{Pbx}$ therapy did not

61 substantially protect against oral dysbiosis or ameliorate cell loss, it did dampen

62 inflammation and $\mathrm{T}$ cell activation. Collectively, these data provide one of the most

63 comprehensive evaluations of SIV-induced changes in oral microbiome and CD4+ T cell

64 populations, and also suggest that oral $\mathrm{Pbx}$ could be a simple therapy to improve anti-

65 inflammatory states in addition to more traditional antivirals. 
69 of mucosal homeostasis, inflammation, loss of CD4+ T cells and translocation of microbial

70 products from the intestines into circulation and lymphoid tissues ${ }^{1-4}$. Circulating

71 lipopolysaccharide (LPS) and SCD14 are established biomarkers for microbial

72 translocation and major modulators of inflammation by engaging TLRs. One proposed

73 methodology to modulate inflammation is by regulating the microbiome of the lower

74 gastrointestinal tract through probiotic supplementation $(\mathrm{Pbx})$. Indeed, $\mathrm{Pbx}$ therapy has

75 previously been used to improve general mucosal function and decrease the incidence of

$76 \mathrm{GI}$ inflammation and disease, specifically inflammatory bowel disease, ulcerative colitis

77 and Crohn's disease ${ }^{5-11}$. Pbx enhance NF-kB signaling and improve epithelial barrier

78 function, likely through TLR signaling ${ }^{12-14}$, and multiple lines of evidence suggest Pbx use

79 in persons living with HIV $(\mathrm{PLWH})$ may reduce microbial translocation and inflammation

$80^{15-18}$. Further studies using SIV infected nonhuman primate models also demonstrate

81 improved immune function, reduced cellular immune activation, and decreased co-morbid

82 conditions ${ }^{19,20}$.

83 Due to a lack of global access to cART and ongoing oral immune activation in

84 PLWH, oral complications in HIV disease are increasingly common. Oral bacterial and 85 mycotic co-infections such as candidiasis, necrotizing gingivitis, and periodontitis are all 86 common in $\mathrm{PLWH}^{21-23}$, as are KSHV and EBV infection of the oral epithelia resulting in

87 increased oral tumors and hairy leukoplakia ${ }^{24-26}$. Much like has been shown in HIV 88 disease, complications of the oral mucosa are also routinely found during experimental

89 SIV infection of macaques. Although the mechanisms are not entirely clear for oral

90 diseases in HIVISIV infections, SIV-induced dysbiosis of the oral microbiome does result

91 in increased production of cytokines and other soluble inflammatory mediators in the oral 
92 cavity, while simultaneously suppressing antimicrobial functions and epithelial

93 development ${ }^{27}$.

94 So called innate lymphoid cells (ILC) are identifiable by their lack of common

95 lineage markers (those identifying T cells, B cells, NK cells, and myeloid cells), and can

96 be distinguished by high expression of the IL-7 receptor, CD127 ${ }^{28-31}$. ILC3 in particular

97 play a major role in modulating gut homeostasis, but can be disrupted by changes in the

98 inflammatory microenvironment, infectious disease, and shifts in the microbiome ${ }^{32}$. HIV

99 and SIV infections drive a perturbation of ILC3 resulting in loss of intestinal barrier integrity,

100 increased inflammation and potential dysregulation of the local microflora. Specifically,

101 others and we have shown that acute SIV infection causes depletion of ILC3 in the GI

102 mucosae ${ }^{33-35}$. In PLWH, ILCs are depleted but partially restored by ART ${ }^{36}$. Much like had

103 been shown in SIV-infected macaque models loss of ILC3 is associated with microbial

104 translocation. Neither HIV nor SIV infection directly depletes ILC3, but rather changes in

105 the cytokine milieu and local microbiotic signals lead to increased apoptosis ${ }^{33,34,36}$.

106 Importantly, we have previously shown that probiotic therapy improves IL-23 production

107 and as a result expands ILC3 numbers in naïve macaques ${ }^{37}$.

108 It has been shown that HIV alters human microbiome including oral bacteria 109 communities ${ }^{38}$. Periodontal disease, a common oral inflammatory infection in HIV infected

110 patients, has been shown to be associated with general dysbiosis in the oral cavity, as

111 well as with HIV disease progression ${ }^{39}$. Dysbiosis in the oral cavity and changes to the

112 oral bacterial communities might play a role in the sustained host proinflammatory

113 responses to the virus. Restorative therapies that could improve oral and GI function,

114 inflammation, and microbial health could be of significant benefit to PLWH. Indeed,

115 previous studies by our groups have demonstrated that therapy with probiotic, beneficial

116 bacteria can improve mucosal health and dampen inflammation in health macaques as

117 well as in the context of SIV infection ${ }^{37,40,41}$. Thus, we hypothesized that probiotic 
118 supplementation prior to SIV infection and during acute infection may enhance the oral

119 microbiome and prevent pathogenic consequences of SIV. In this study we evaluated the

120 effects of pre- and acute administration of oral probiotic therapy on viral kinetics,

121 inflammation, CD4+ T cell loss, microbiome, and ILC3 modulation in the oral and GI

122 mucosae, and associated lymphoid tissues in SIV-infected rhesus macaques.

123 


\section{RESULTS and DISCUSSION}

\section{Early probiotic administration during SIV infection}

126 To determine the effects of probiotic $(\mathrm{Pbx})$ administration pre-SIV and during acute SIV

127 infection, we evaluated 12 Indian origin rhesus macaques, 6 receiving oral $\mathrm{Pbx}$ and 6

128 receiving oral vehicle control. Animals were given $\mathrm{Pbx}$ for a total of 42 days: 28 days pre-

129 virus challenge and for a subsequent 14 days following challenge (Figure 1A). Macaques

130 were intrarectally challenged with SIVmac251 as described previously ${ }^{42}$, and buccal,

131 colorectal, and lymph node biopsies in addition to blood draws were collected as indicated.

132 No significant differences were observed in plasma viral loads longitudinally (Figure 1B-

133 C), nor were any differences observed in peak viral loads between the two groups (Figure

134 1D). However, previous studies have also reported that the benefits of probiotic therapy

135 in HIV an SIV are uncoupled from viremia which is generally not affected ${ }^{40}$.

\section{T cell responses during Pbx treatment of SIV-infected macaques}

138 To assess broad antiviral T cell responses we performed longitudinal ELISPOT assays on

139 PBMC as well as tissues at necropsy. Interestingly, Pbx-treated animals consistently had

140 lower levels of both Gag (4-fold at day 112) and Env (9-fold at Day 112) ELISPOT

141 responses (Figure 2A-B). Further, the total magnitude of the ELISPOT response (as

142 measured Pol, Env, Gag) in control animals was significantly greater at necropsy in all

143 tissues measured (PBMC, spleen, oral lymph nodes, and tonsil).

144 Since SIV infection has a significant impact on memory CD4+ T cell populations,

145 as well as CD8+ T cell activation, we next used polychromatic flow cytometry to evaluate

146 these cell subsets in detail in Pbx-treated animals. As expected, the frequency of total

147 CD4+ T cells in circulation declined early following SIV challenge concomitant with an

148 increase in the frequency of CD8+ T cells (Figure 3A). Similar trends were observed in

149 colorectal tissue where the shift was even more robust (Figure 3B), in line with previous 
150 studies ${ }^{43,44}$. Remarkably, this study demonstrated for the first time an acute and sustained

151 loss of CD4+ T cells in the buccal mucosa following SIV infection (Figure 3C). However,

152 little to no difference was observed in CD4+ and CD8+ T cell dynamics in any tissue when

153 comparing Pbx-treated to control animals. There was also little difference between the

154 two groups when specifically examining CD28+CD95+ central memory CD4+ T cells in

155 any tissue (Figure 4A). However, Pbx treatment reduced Ki67 expression (measured at

156 day $42 \mathrm{Pbx} /$ Day 14 post-challenge) in both bulk and CM CD4+ T cells in most tissues

157 (Figure 4B). This suggests that while Pbx may not protect CD4+ T cell loss, it may reduce

158 T cell activation, which is well in line with previous observations ${ }^{37}$. These data indicate

159 Pbx could be a beneficial augmentive therapy to protect CD4+ T cells and reduce overall

160 cellular activation.

\section{Effects of probiotic treatment on ILC3 populations during SIV infection}

163 We and others have previously shown that HIV and SIV infection can modulate ILC3

164 populations in the mucosae. Further, our groups have demonstrated that probiotic

165 supplementation can actually expand ILC3 numbers in some animals and could possibly

166 function as a putative rheostat mechanism of action for controlling inflammation during

167 lentivirus infections. In this study we quantified the frequency of Lin-NKp44+NKG2A/C-

168 ILC3, as described previously ${ }^{34,45}$, in longitudinal colorectal and buccal biopsies.

169 Interestingly, little change was observed in ILC3 frequencies during the probiotic

170 supplementation period (day 0 to day 28 ) in this study (Figure 5). Although some animals

171 demonstrated minor transient increases in ILC3 day 14 (colorectal) or day 28 (oral)

172 following probiotic supplementation, these changes were not significant. It is unclear why

173 ILC3 might not be expanded in this context, but previous work from our laboratory has

174 shown that colorectal ILC3 are less likely to be increased compared to those in the jejunum

175 and there is significant animal-to-animal variation ${ }^{37}$. Further, $\mathrm{Pbx}$ only appeared to 
176 marginally impact SIV-induced changes in ILC3 frequencies, indicating that while Pbx may

177 impact T cell responses, it is unlikely to protect against loss of ILC3.

179 Soluble measures of inflammation and immune activation

180 To further address the impact that Pbx and ILC3 modulation might be having on global

181 inflammation during SIV infection we compared soluble inflammatory mediators in the

182 circulation by Luminex. Animals treated with $\mathrm{Pbx}$ had a distinctly lower inflammatory

183 profile on day 28 (day of challenge), suggesting that Pbx treatment alone was sufficient to

184 decrease soluble mediators of inflammation (Figure 6A). This reduced inflammatory

185 profile persisted from day 42 (day 14 post-challenge), and even persisted in some animals

186 up to day 112 (day 84 post-challenge. However, Pbx therapy did not appear to have any

187 effect on microbial translocation products (Figure 6B), which is similar to HIV studies

188 showing improved immune function but no consistent decline in circulating bacterial 189 products ${ }^{46,47}$.

191 Probiotic supplementation partially modulates oral microbiome during SIV infection

192 To study the effects of Pbx on the microbiome, we used 16s rRNA gene sequencing to

193 profile bacterial communities in the buccal and colonic mucosa. No notable differences in

194 community richness, evenness, Shannon diversity or over all principal components

195 analysis differences were observed between groups in either oral or gut samples,

196 consistent with previous studies of probiotic supplementation. The most abundant

197 bacteria in the oral mucosa of all animals were of Firmicutes phyla, with lesser

198 representation of Bacteroidetes, Proteobacteria, Fusobacteria, and Actinobacteria (not

199 shown). The colorectal mucosa top phlya was Epsilonbacteraeota followed by

200 Bacteroidetes and Spirochaetes (not shown). At the phyla level, no notable differences

201 between groups or across time points were observed in either site. At the genus level for 
202 the oral mucosa, a high abundance of Streptococcus and Veillonella was observed in all

203 samples, followed by minor abundances of Alloprevotella, Carnobacterium, Actinobacillus,

204 Porphyromonas, Haemophilus, Gemella, and Prevotella (Figure 7A). The top genera for

205 colorectal samples were dominated by Helicobacter with an abundance of fifty-five percent

206 followed by Prevotella, Treponema, Alloprevotella, Ruminococcaceae, Camplobacter with

207 abundances starting at nine percent (Figure 7B). We performed differential abundance

208 analysis on the Pbx and placebo groups across all timepoints in the oral mucosae. In the

209 placebo group, Porphyromonas and Actinobacillus were found to be significant between

210 day -21 and $0(p=0.012$ and 0.021$)$, day -21 and day $28(p=0.028)$, and day 28 and day

$21142(p=0.004$ and 0.001) (Figure 7C-D). In the Pbx group, Porphyromonas and

212 Actinobacillus were significant between day -21 and day $28(p=0.04)$ and day 42 and day

$213112(p=0.037$ and $p=0.017)$ (Figure 7D). However, notable differences were not observed

214 between groups, however it does appear that the changes observed were dampened in

215 the probiotics group compared to the placebo group. Thus, while probiotics did not have

216 a substantial impact in modulation of the microbiome, these changes reflect possible

217 dampening of SIV-mediated dysbiosis in the oral microbiome.

\section{Concluding remarks}

220 In summary our study presents a number of new findings relevant to SIV immunobiology.

221 First, SIV infection clearly demonstrates an early depletion of CD4+ memory T cells in the

222 oral mucosa. Next, Pbx does not appear to protect against loss of CD4+ T cells or ILC3

223 but does reduce measures of systemic inflammation. These data also offer one of the

224 most in-depth characterizations of microbiome in the oral mucosae, where we

225 demonstrated novel changes to the oral microbiome after SIV infection, which are slightly

226 dampened with probiotics. However, no substantial microbiome alterations were observed

227 between probiotics and placebo groups. Overall, our studies indicate a role for oral $\mathrm{Pbx}$ 
bioRxiv preprint doi: https://doi.org/10.1101/2020.12.17.423321; this version posted December 18, 2020. The copyright holder for this preprint (which was not certified by peer review) is the author/funder. All rights reserved. No reuse allowed without permission.

228 in modulating SIV-induced inflammation, but additional work will be required to tease out 229 detailed mechanisms of action.

230 


\section{METHODS}

\section{Ethics statement}

233 All animals were housed at Biomere, Inc., Worcester, MA in accordance with the rules and

234 regulations of the Committee on the Care and Use of Laboratory Animal Resources.

235 Animals were kept in agreement with the American Association for Accreditation of

236 Laboratory Animal Care standards and The Guide for the Care and Use of Laboratory

237 Animals ${ }^{39}$. Animals were fed standard monkey chow diet supplemented daily with fruit

238 and vegetables and water ad libitum. Social enrichment was delivered and overseen by

239 veterinary staff and overall animal health was monitored daily. Animals showing significant

240 signs of weight loss, disease or distress were evaluated clinically and then provided

241 dietary supplementation, analgesics and/or therapeutics as necessary. Animals were

242 humanely euthanized using an overdose of barbiturates according to the guidelines of the

243 American Veterinary Medical Association. All studies reported here were performed under

244 IACUC protocols 17-02 and 16-08 which was reviewed and approved by the Biomere

245 IACUC. When necessary, macaques were immobilized with ketamine $\mathrm{HCl}$ (Parke-Davis)

246 at approximately $10 \mathrm{mg} / \mathrm{kg}$ and injected intramuscularly after overnight fasting. Blood

247 samples were collected using venipuncture.

\section{Animals, probiotic supplementation and SIV challenge}

250 Sixteen experimentally naive age-matched Indian-origin rhesus macaques (Macaca

251 mulatta) were analyzed in this study. All animals were colony-housed at Biomere, Inc. and

252 were free of simian retrovirus type $D$ and simian T-lymphotropic virus type 1. Animals

253 were challenged intrarectally with SIVmac251 as described previously ${ }^{42}$. Six animals

254 received Visbiome for 42 days mixed with food, while six received food only (vehicle)

255 control. No adverse events due to Pbx therapy were observed in any of the animals.

\section{Blood and tissue processing}


258 Blood samples were collected in EDTA-treated tubes, and peripheral mononuclear cells

259 (PBMCs) were isolated using standard density gradient centrifugation over lymphocyte

260 separation media (MP Biomedicals, Solon, OH) and any contaminating red blood cells

261 were lysed using hypotonic ammonium chloride solution. Processing of tissues was

262 carried out using protocols optimized in our laboratory ${ }^{27,30,41}$. Briefly, colon and buccal

263 tissue were cut into $1 \mathrm{~cm}^{2}$ then incubated in 5mM EDTA for 30 min before undergoing

264 mechanical and enzymatic disruption. Samples were then gently pushed through filters

265 before lymphocytes were isolated via bilayer (35\%/60\%) isotonic Percoll density gradient.

266 Lymph nodes and spleen were trimmed of excess tissue then mechanically disrupted.

267 Manual cell counts were performed for each sample using Trypan Blue. Two million cells

268 from each sample were then used for real-time flow cytometry staining while remaining

269 cells were cryopreserved in a DMSO solution and stored in liquid nitrogen vapor.

271 ELISPOT

272 ELISPOTs were performed with Mabtech IFN-y ELISpot Plus Kits. Peptides used were

273 SIVmac239 Gag (Cat: 12364), ENV (Cat: 12635), and POL (Cat: 12766) obtained through

274 the NIH AIDS Reagent Repository and aliquoted into pools. Briefly, samples were thawed

275 and rested for four hours. Cells were then counted and aliquoted into 96 well plates at

$2760.25 \mathrm{M}-0.2 \mathrm{M}$ cells per well. Following incubation at 37 degree $\mathrm{C}$ and substrate

277 development, plates were read by ZellNet Consulting (Fort Lee, NJ).

\section{Antibodies and flow cytometry}

280 Flow cytometry staining of mononuclear cells was carried out for cell surface and 281 intracellular molecules (Supplementary Table 1). Briefly, samples were incubated with

282 the LIVE/DEAD Aqua amine dye (Invitrogen, Carlsbad, CA), then washed before staining 283 with surface antibodies. After staining, cells were permeabilized using Thermo Fisher 
284 Scientific Fix \& Perm buffer kit (ThermoFisher Scientific, Waltham, MA) and incubated with

285 the intracellular staining antibodies. Isotype-matched controls were included in all assays.

286 All acquisitions were made on an LSRII (BD Biosciences) and analyzed using FlowJo 287 (9.9.6).

289 Viral load quantification

290 Quantified RNA was used for RT-PCR against a conserved region of gag using gene-

291 specific primers ${ }^{48}$. In the first step, RNA was reverse-transcribed followed by treatment

292 with RNase for 20 min at $37^{\circ} \mathrm{C}$. Next, cDNA was amplified using 7300 ABI Real-Time PCR

293 system (applied Biosystems) according to the manufacturer's protocol.

\section{DNA extraction, 16S rRNA gene sequencing and data analysis}

296 We extracted DNA from cryopreserved buccal and colorectal biopsies using the

297 PowerFecal DNA Isolation Kit (Qiagen, Valencia, CA). We then prepared sequencing 298 libraries as described by the Earth Microbiome Project and sequenced them using the 299 Illumina MiSeq Sequencer (Illumina, San Diego, CA). 515F-806R primers were used to 300 sequence the V3-V4 region of the 16 s SSU rRNA as previously described ${ }^{49}$. 16 s 301 sequencing reads were demultiplexed, trimmed and then processed using QIIME2 302 (version 2020.2) ${ }^{50}$. Operational taxonomic units (OTUs) were clustered at 99\% similarity 303 using the DADA2 method and assigned taxonomy with the Silva 132 classifier for 304 taxonomic determination. All sequence reads and operational taxonomic unit (OTU) 305 observations were included in our analyses, in order to maximize the observed diversity 306 of the bacterial communities and was done utilizing $R$ (v3.6.0) packages. Alpha diversity 307 (richness, evenness (Pielou)), and Shannon diversity) was calculated by the microbiome 308 package, beta diversity was calculated with pairwise sample dissimilarity and unweighted 309 unifrac ordination analysis by means of principal coordinates analysis (PCoA) using the 
310 vegan package for Ordination, Diversity and Dissimilarities. Statistical analyses were

311 completed using the Pairwise Adonis package. Differential abundance analysis was

312 completed using DESeq2. Beta diversity and taxonomic plots were created utilizing the

313 phyloseq and ggplot. Alpha diversity and differential abundance plots were created in

314 Prism 8 version 8.4.3 (GraphPad Software).

\section{Statistical analyses}

317 Statistical analyses were carried out using Prism Version 8.3.0 (GraphPad Software).

318 Unpaired, nonparametric, Mann-Whitney $U$ tests, Wilcoxon, and $t$ tests were used where

319 indicated, and $\mathrm{P}<0.05$ were assumed to be significant. Samples were grouped and

320 heatmaps were generated in Prism version 7.0d (GraphPad Software). For beta diversity,

321 statistical significance was assessed using the pairwise permutational multivariate

322 analysis of variance (PERMANOVA; Adonis) test in R. For taxonomic differential

323 abundance DESeq2 was used and accounted for sequencing depth and multiple

324 comparisons using the Benjamini and Hochberg method to control the false discovery rate

325 (FDR) and adjusted $\mathrm{p}$ values of $<0.05$ were considered significant.

\section{Multiplex cytokine analysis of plasma}

328 Plasma samples were analyzed for rhesus macaque cytokines and chemokines by

329 Luminex using established protocols for non-human primates. Evaluation of analytes IL-

330 6, IL-12p70, IL-17, TNF- $\alpha$, IL-2, G-CSF, CXCL13, MIP-1 $\beta$, IP-10, IL-1Ra, Perforin, IFN- $\alpha$,

331 IFN- $\gamma$, MCP-1, MIG, II-23 and MIF were included in this assay. Only analytes quantifiable

332 above the limit of detection in plasma are presented. Log2 fold change for Luminex data

333 was calculated per RM in this study. Briefly, each rhesus macaque timepoint log2 fold

334 change was calculated using the following formula: 
336 Log2 ( Day \# analyte level / Day 0 analyte level )

338 NaN or Inf values were replaced with NA. Log2 fold change was calculated in R v4.0.0

339 (https://www.R-project.org) and heatmaps were generated with pheatmap v. 1.0.12

340 (https://CRAN.R-project.org/package=pheatmap). Row clustering was performed on

341 cytokine log2 fold changes with default parameters. Column clustering was not performed.

342

343 


\section{Acknowledgements}

345 The authors thank Michelle Lifton, Dr. James Whitney, and Dr. So-yon Lim for technical

346 assistance, Dr. Dan Barouch for providing the SIV challenge stock, as well as Dr. Luis

347 Giavedoni (Southwest National Primate Research Center) for assistance with Luminex

348 assays. We also thank Dr. Angela Carville and Biomere, Inc. for animal management.

\section{Conflicts of interest}

351 All authors report no financial conflicts of interest.

\section{Author contributions}

354 R.K.R and N.K. designed the project. R.J., S.S., K.K., V.V., B.H., S.V.S., D.R., and C.M

355 processed samples. V.V. coordinated animal studies, and R.J., K.K., C.B., L.S., and

356 R.K.R. analyzed the data. All authors contributed to the final preparation of the manuscript. 
360 Figure 1. Oral Pbx therapy and SIVmac251 challenge in rhesus macaques. Timeline of study protocol (A); Pbx (blue) and vehicle control (red) groups. Both groups were challenged intrarectally with SIVmac251 at day 28. Tissue collections and treatment duration are indicated. Longitudinal viral loads (B) for individual animals, mean longitudinal (C) viral loads, and peak viral loads (D) of probiotic and control groups are quantified as described in the Methods.

Figure 2. ELISPOT responses following SIV challenge. Mean spot forming cells using GAG (A) and ENV (B) peptide pools analyzed longitudinally following SIVmac251 infection. PBMC, spleen, oral lymph node, and tonsil mean spot forming cells (C) from probiotic and control animals are shown for POL (blue), ENV (red), GAG (green) ELISPOTS at necropsy. Unpaired $t$ tests were used to compare total ELISPOT values between groups; *, $\mathrm{P}<0.05$.

Figure 3. Bulk T cell analyses following Pbx therapy and SIV challenge. Longitudinal frequencies of CD4+ and CD8+ T cells in PBMC (A), colorectal (B), buccal (C), and PLN biopsies (D) are compared between control (red) and Pbx groups (blue). PLN, peripheral lymph nodes.

Figure 4. Memory CD4+ T cell analyses following Pbx therapy and SIV challenge. Central memory CD4+ T cells longitudinal frequency (A) for PBMC, colorectal, peripheral lymph node (PLN), and buccal, comparison of probiotic and control groups. Expression of $\mathrm{Ki}-67$ on CD4+T cells and central memory $\mathrm{T}$ cells $(\mathrm{B})$ between probiotic and control groups. Unpaired $t$ tests were used to compare groups; ${ }^{*}, \mathrm{P}<0.05 ;{ }^{* *}, \mathrm{P}<0.01$.

Figure 5. ILC3 analyses following Pbx therapy and SIV challenge. ILC3 frequencies were quantified in colorectal (a) and buccal (b) biopsies are multiple timepoints (relative to PBX initiation) and during SIV infection (d28 challenge).

Figure 6. Soluble measures of inflammation. (A) Heatmaps of Luminex data of inflammatory cytokines present in both control and probiotic groups at days 28, 42, and 112 shown as log2 fold changes over baseline values. Columns correspond to individual macaques included in this study. (B) Microbial translocation products post infection of individual animals receiving placebos (red) and probiotics (blue) (B) taken at day 42 and 112.

Figure 7. Microbiome alterations following Pbx therapy and SIV challenge.

(A) Taxonomic barplot of average relative abundance for top 15 genera level bacteria separated by experimental group across timepoints for buccal biopsies. (B) Taxonomic barplot of average relative abundance for top 15 genera level bacteria separated by experimental group across timepoints for colorectal biopsies. (C) Scatter plot of relative abundance of Porphyromonas for buccal biopsies separated by experimental group (red is placebo and blue are probiotics) across all timepoints. DESeq2 was used for statistical assessment and adjusted $p$ values of $<0.05$ significant. (D) Scatter plot of relative abundance of Actinobacillus for buccal biopsies separated by experimental group (red is placebo and blue are probiotics) across all timepoints across timepoints. DESeq2 was 406 used for statistical assessment and adjusted $p$ values of $<0.05$ significant. 
bioRxiv preprint doi: https://doi.org/10.1101/2020.12.17.423321; this version posted December 18,2020 . The copyright holder for this preprint (which was not certified by peer review) is the author/funder. All rights reserved. No reuse allowed without permission.

\section{Figure 1}
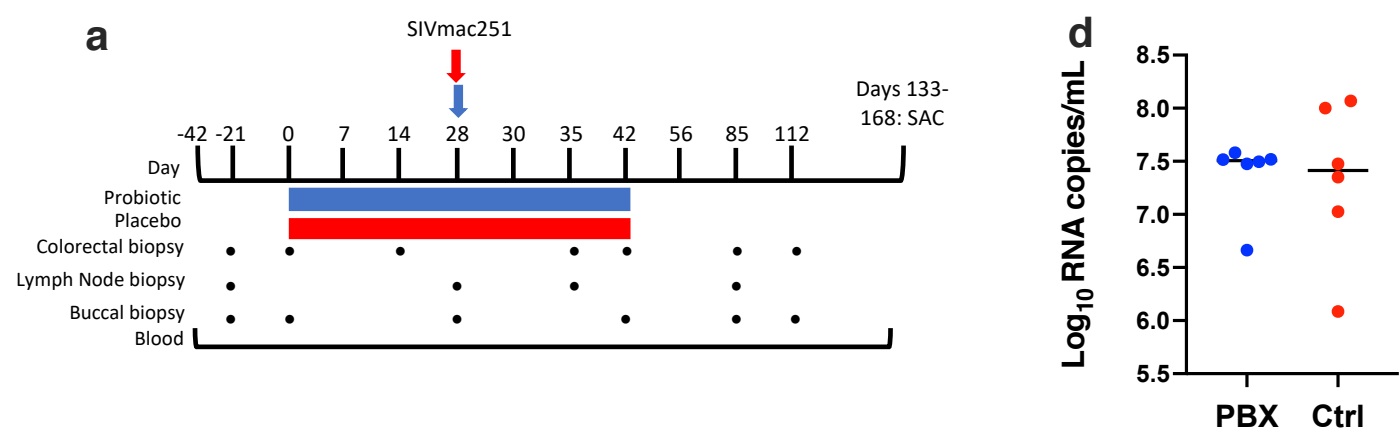

b

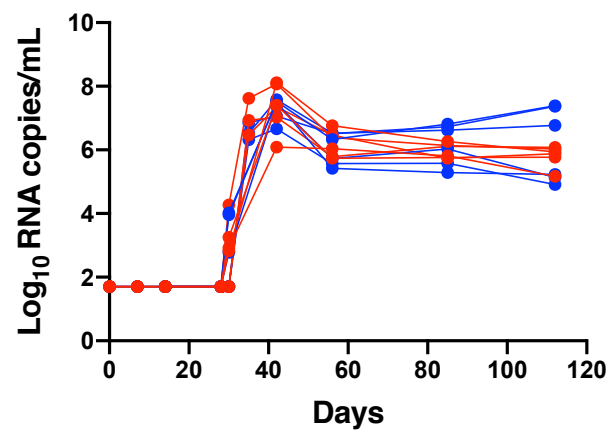

C

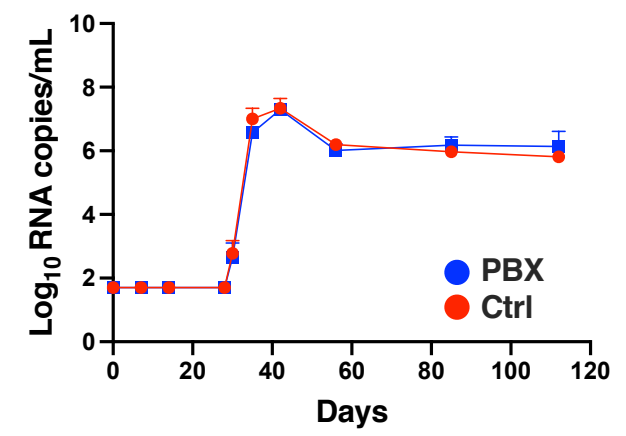

409 

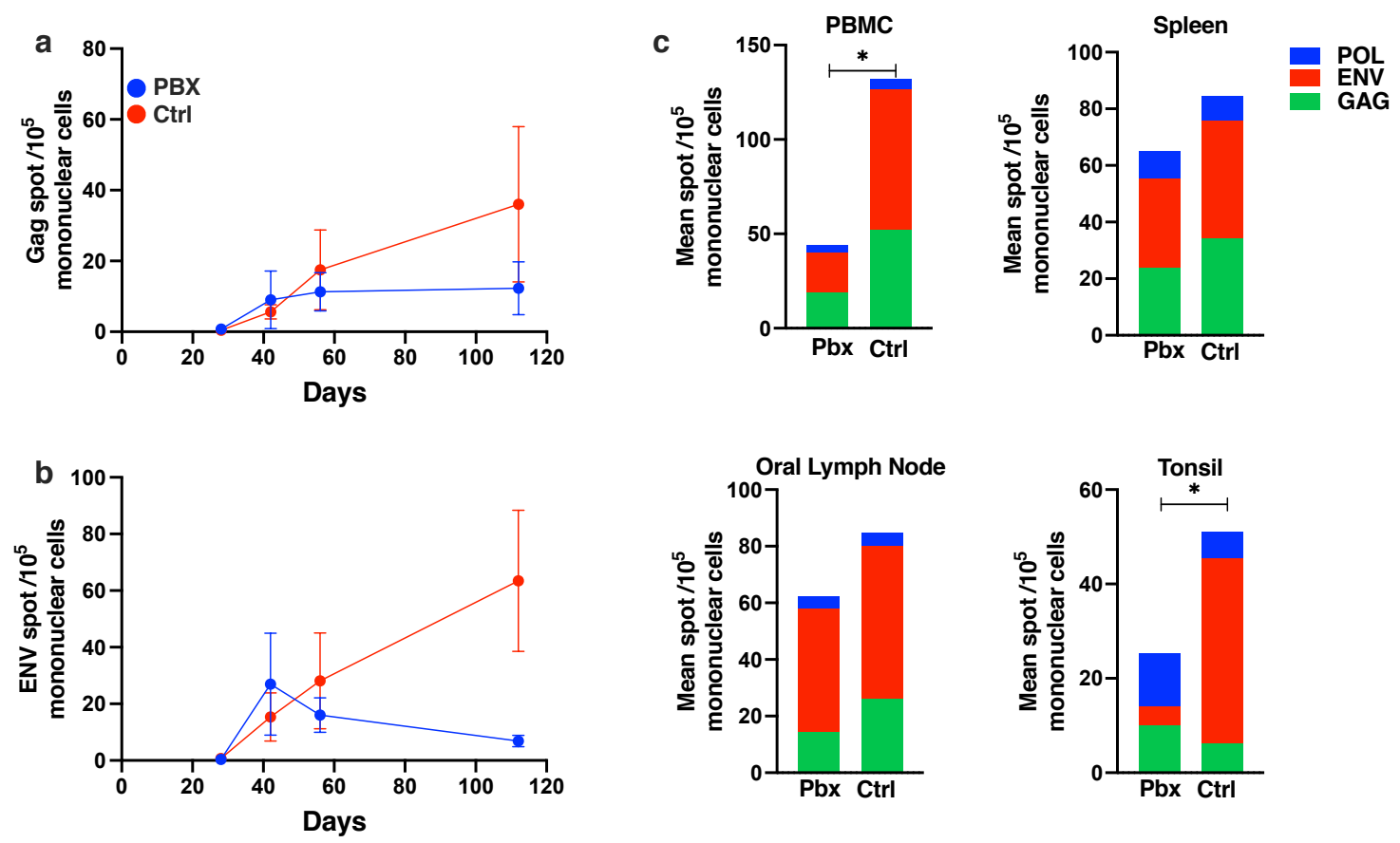


\section{$414 \quad$ Figure 3}
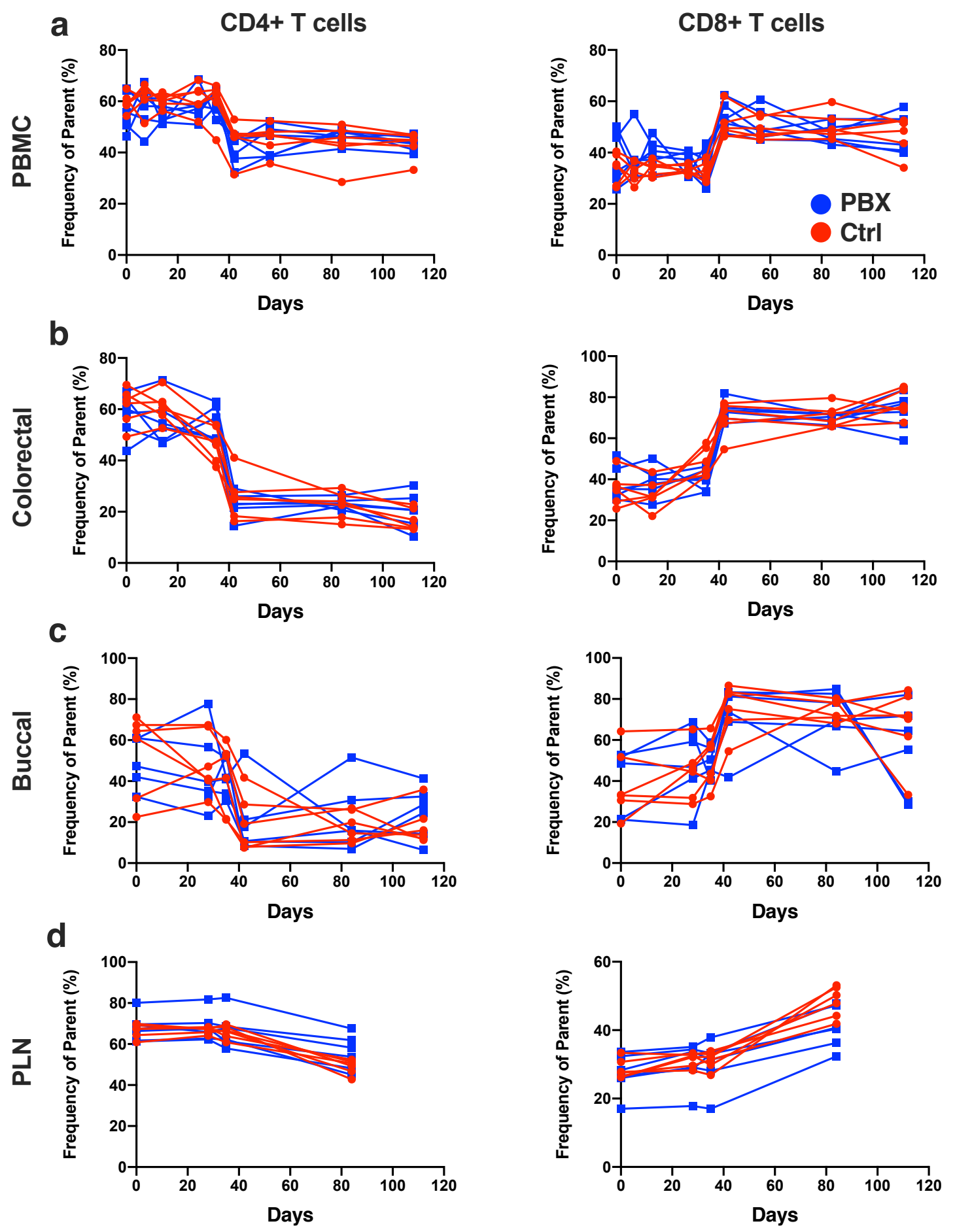

415

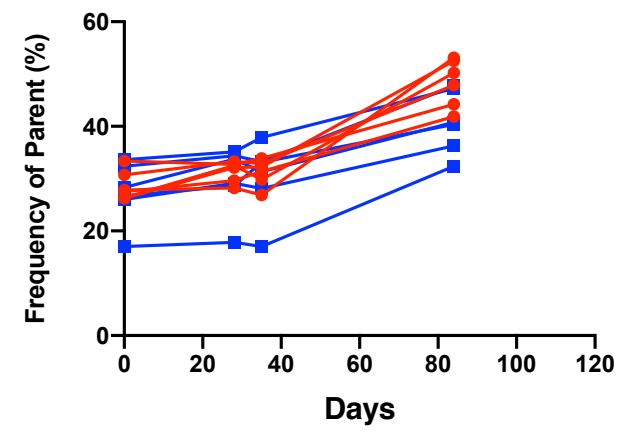




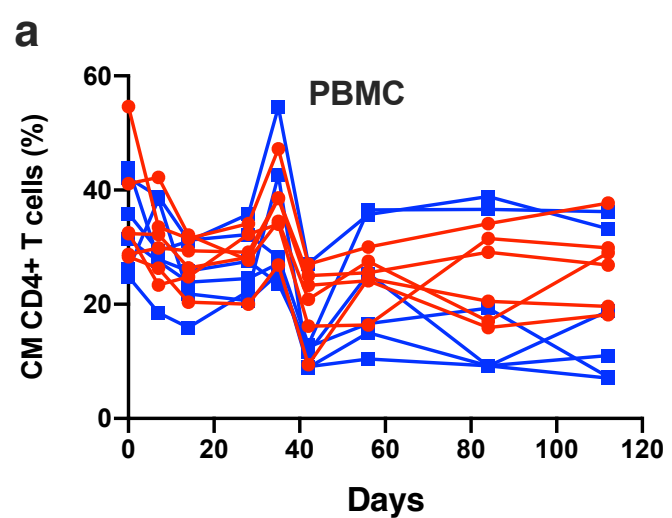

PLN

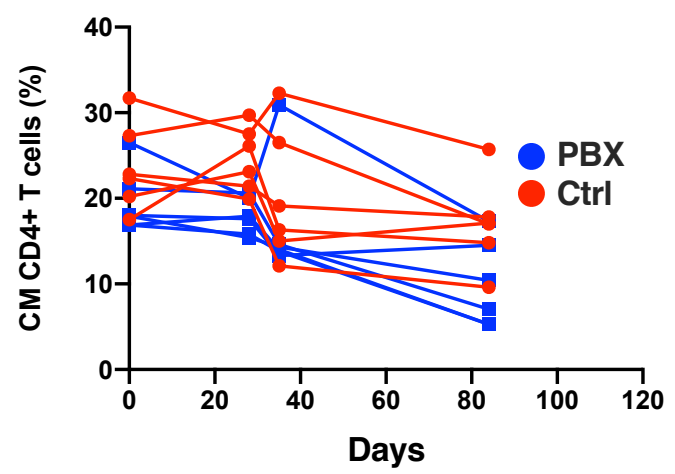

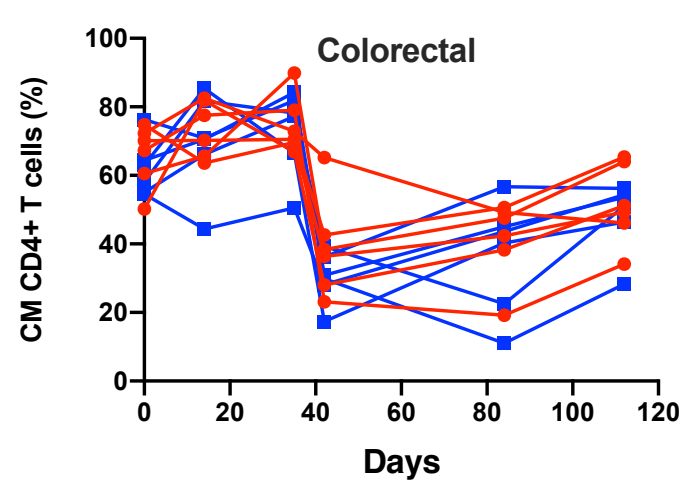

Buccal

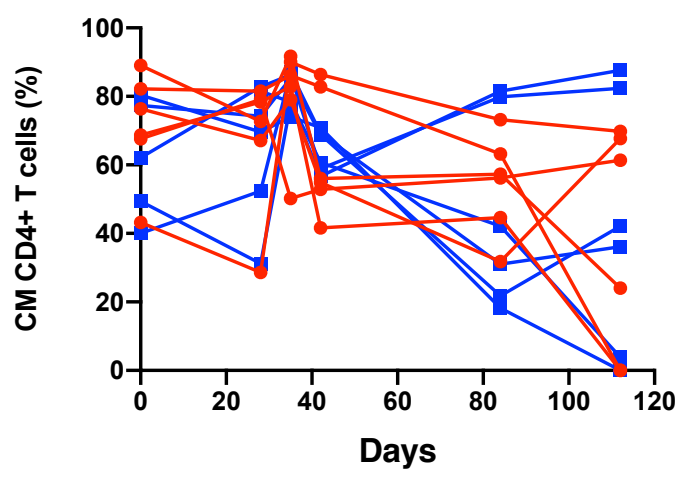

b
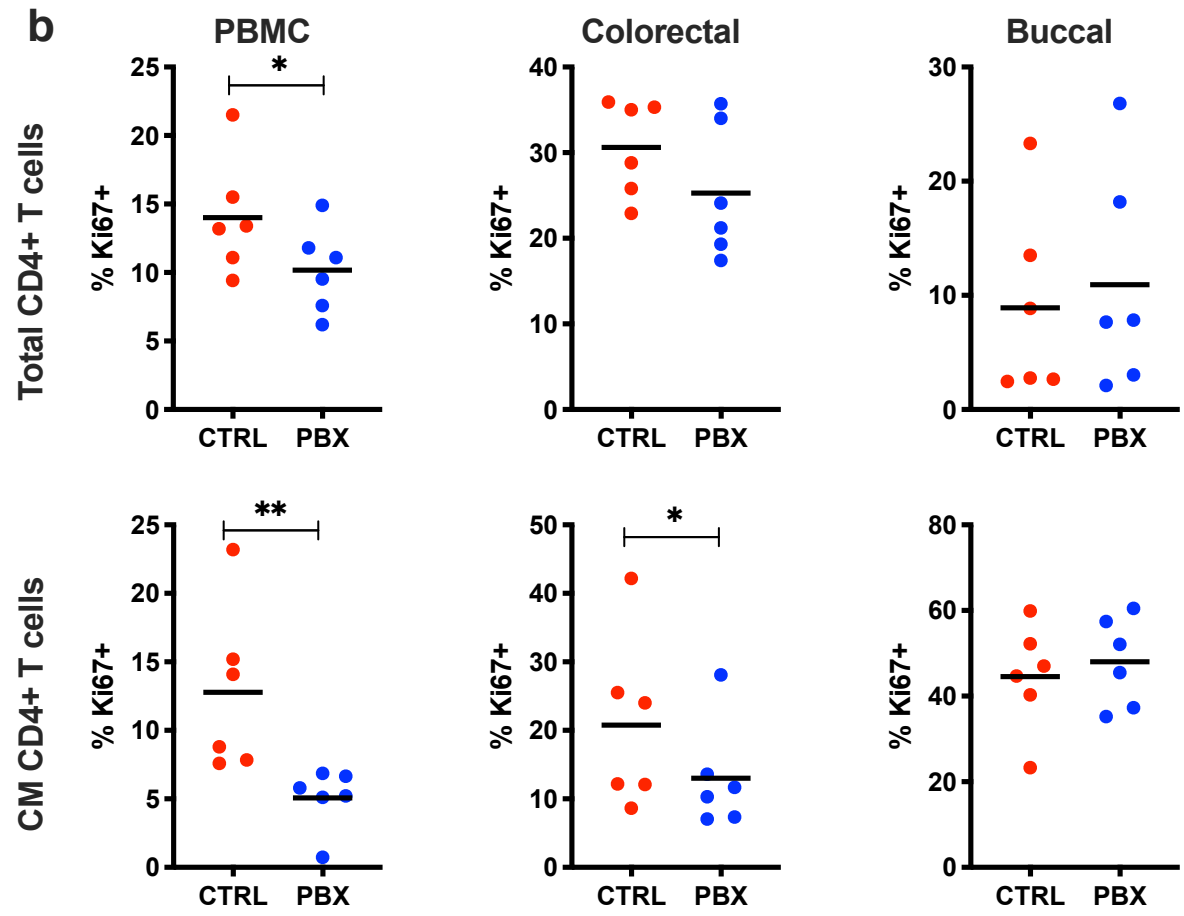
$419 \quad$ Figure 5
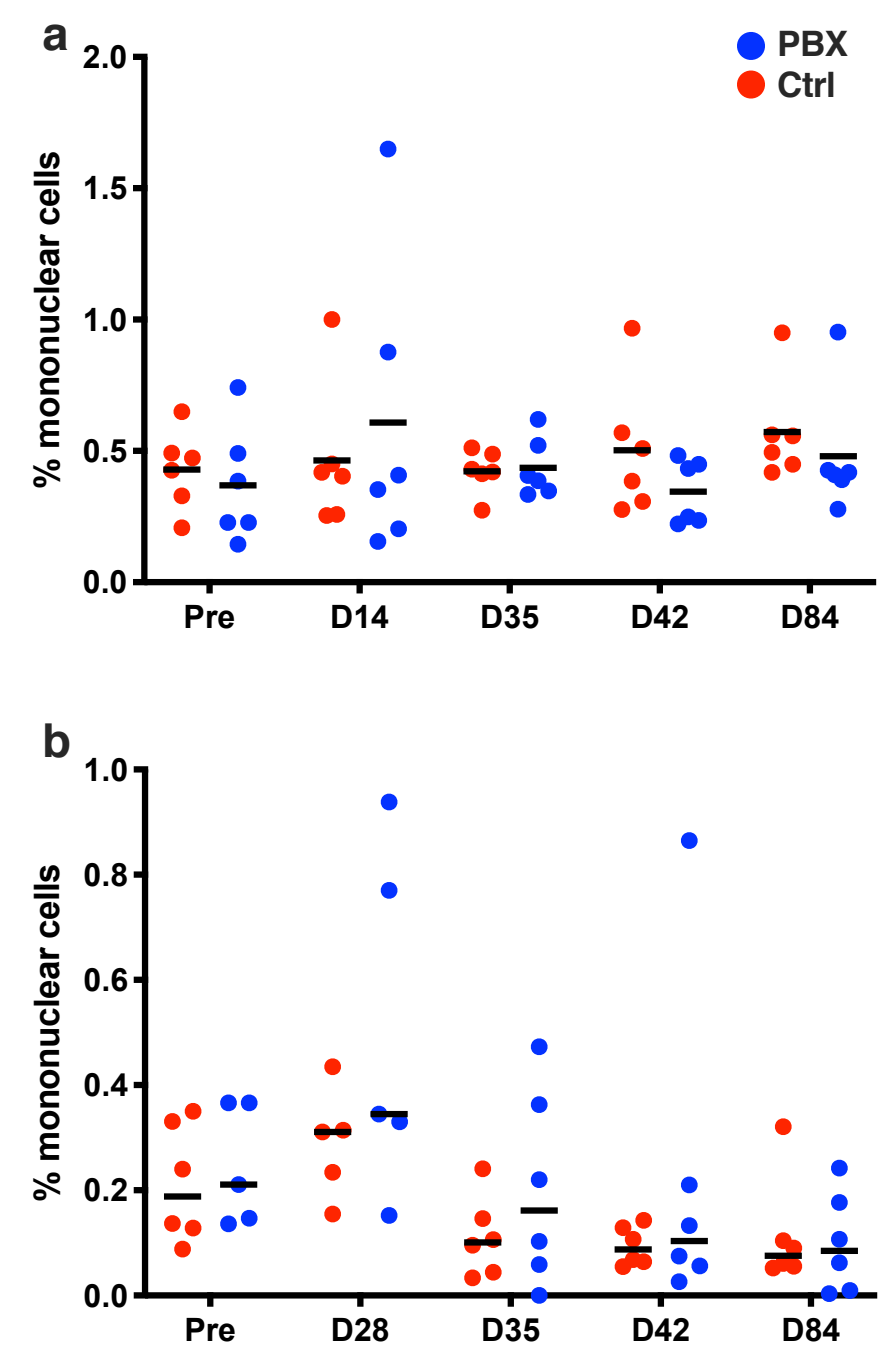


\section{$421 \quad$ Figure 6}

422

a

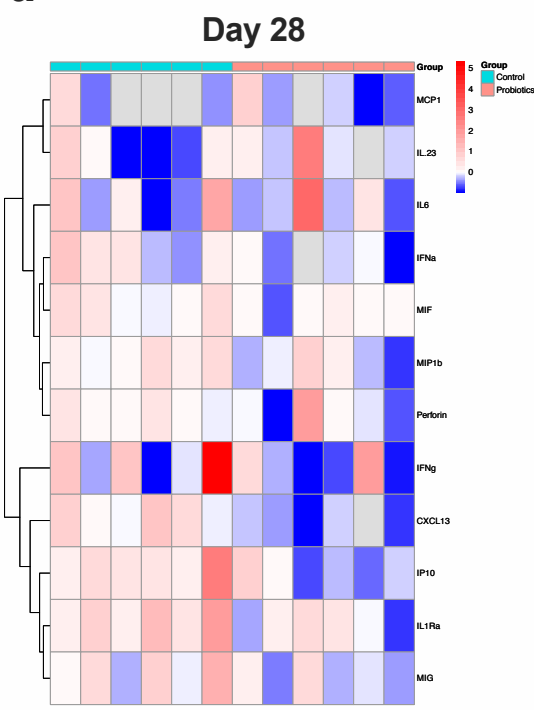

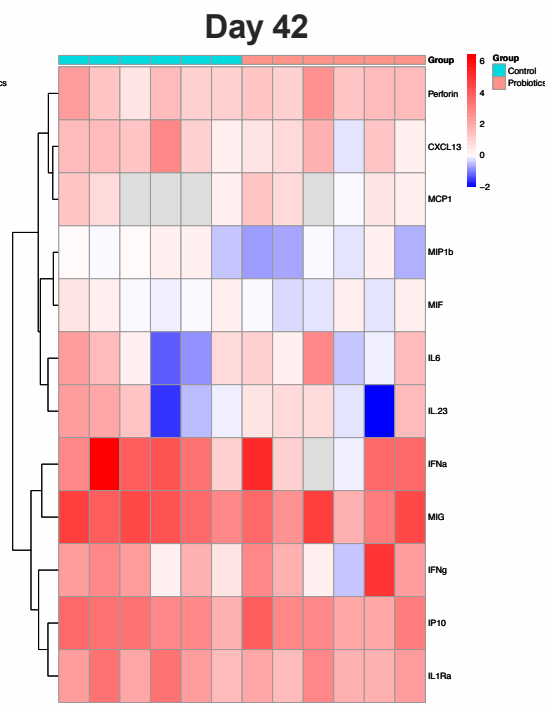

b

Day 42

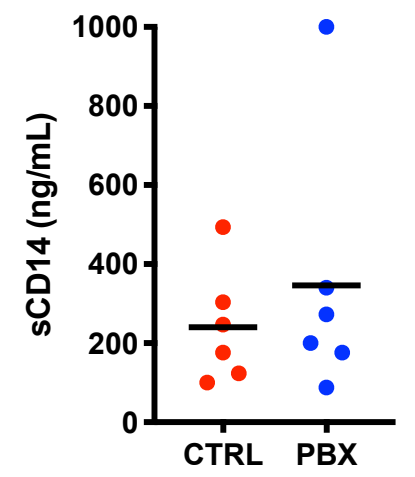

Day 112

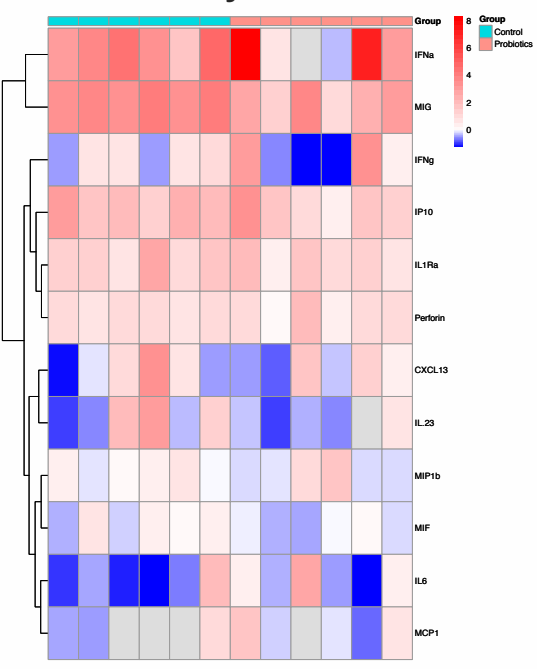

Day 112

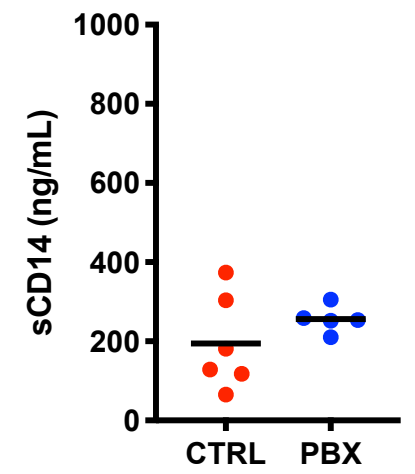


bioRxiv preprint doi: https://doi.org/10.1101/2020.12.17.423321; this version posted December 18, 2020. The copyright holder for this preprint (which was not certified by peer review) is the author/funder. All rights reserved. No reuse allowed without permission.

\section{$424 \quad$ Figure 7}

425

426

427 a

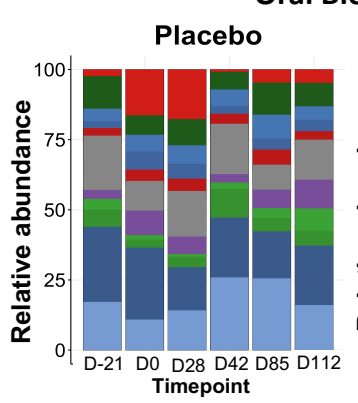

Oral Biopsy: Genera

Probiotics

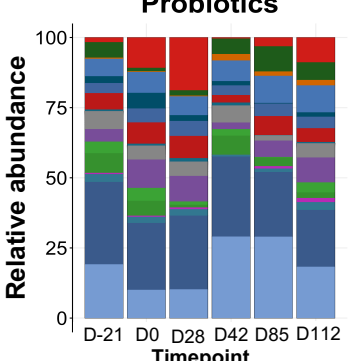

Timepoint
C

Genus

Actinobacillus

Alloprevotella

Carnobacterium

Fusobacterium

Gemella

Leptotrichia

Other

Porphyromonas

Prevotella 7

Prevotella 7
SR1 bacterium oral taxon 875

Streptobacillus

Streptococcus

b

Colorectal Biopsy: Genera
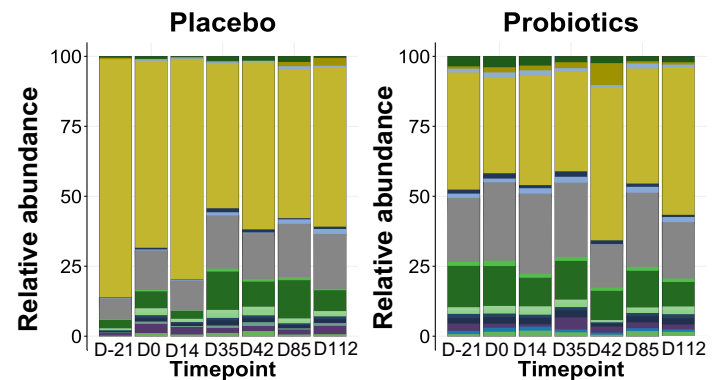

d

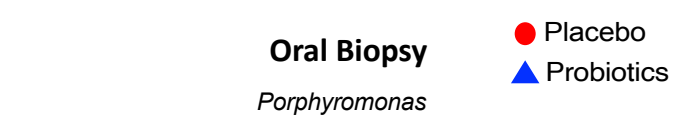

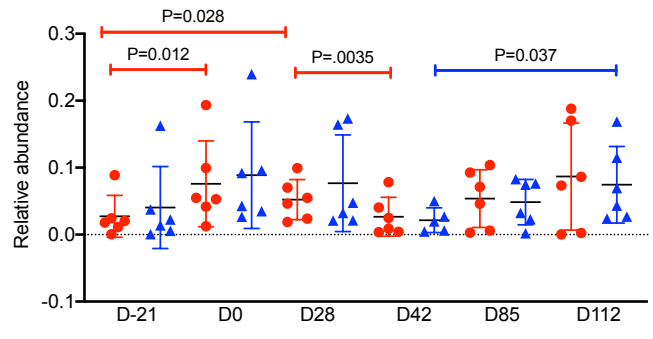

Oral Biopsy

Actinobacillus

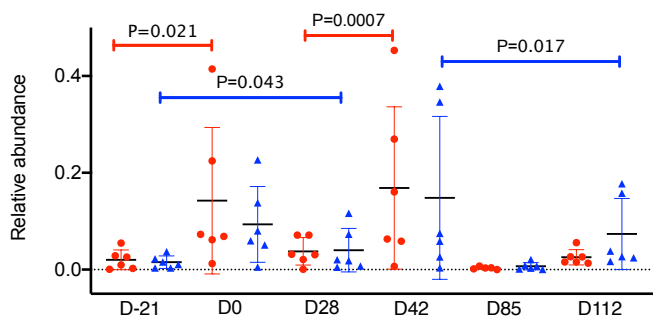



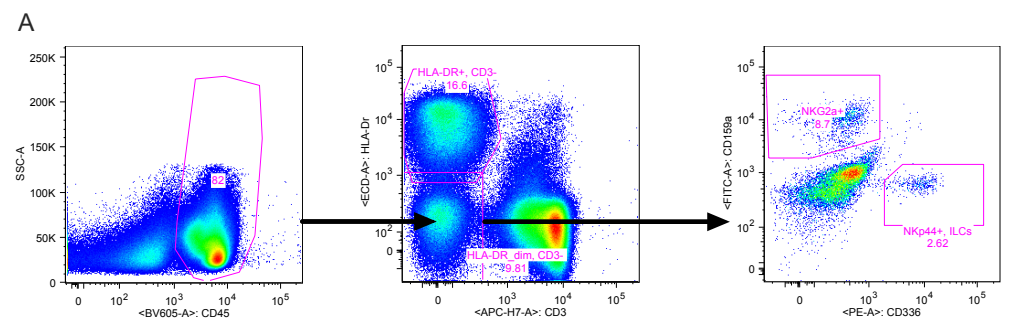

C

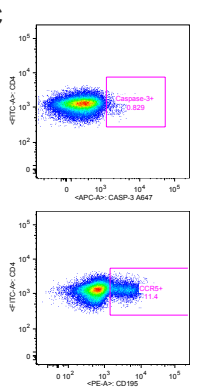

B
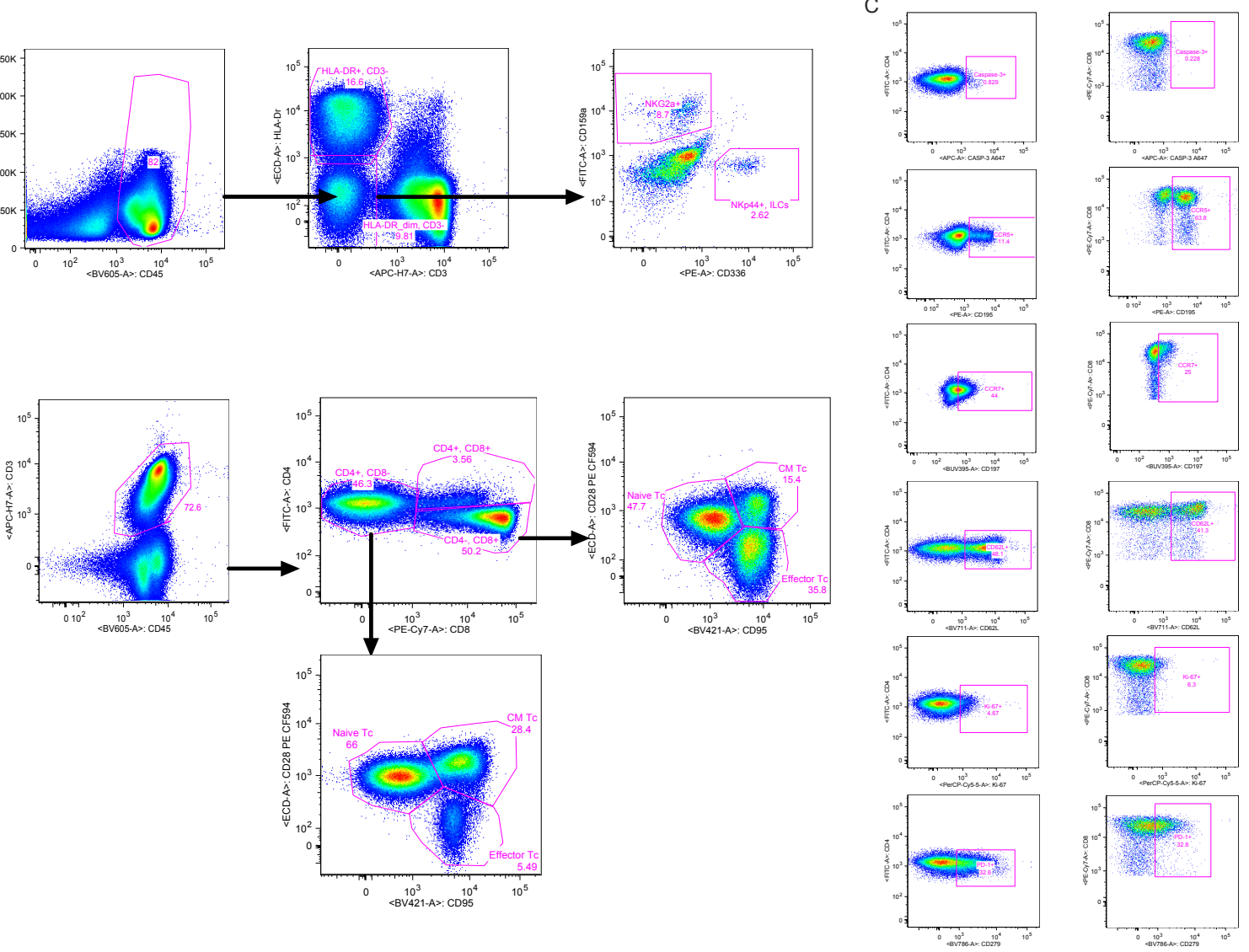

430

431

432

433

434

435

Supplementary Figure 1. Representative flow cytometry gating.

Representative gating strategies are shown for (A) NKG2A/C+ NK cells and NKp44+ ILC3, (B) T cells, and (C) T cell phenotypes. 


\section{Supplementary Table 1}

437

\begin{tabular}{|c|c|c|c|}
\hline $\begin{array}{r}\text { Target } \\
\text { Caspase-3 }\end{array}$ & $\begin{array}{l}\text { Fluorochrome } \\
\text { Alexa647 }\end{array}$ & $\begin{array}{l}\text { Clone } \\
\text { C92-605 }\end{array}$ & $\begin{array}{l}\text { Vendor } \\
\text { BD Pharmingen }\end{array}$ \\
\hline CD3 & APC CY7 & SP34.2 & BD Pharmingen \\
\hline CD4 & FITC & L200 & BD Pharmingen \\
\hline CD8 & PAC BLUE & RPA-T8 & BD Pharmingen \\
\hline CD8 & PE CY7 & RPA-T8 & BD Pharmingen \\
\hline CD11C & A700 & 3.9 & Affymetrix \\
\hline CD14 & BV650 & M5E2 & BD Pharmingen \\
\hline CD16 & BUV496 & $3 G 8$ & BD Pharmingen \\
\hline CD20 & BUV395 & $\mathrm{L} 27$ & BD Pharmingen \\
\hline CD28 & PE CF594 & 28.2 & BD Pharmingen \\
\hline CD45 & BV605 & D058-1283 & BD Pharmingen \\
\hline CD56 & BV786 & NCAM16.2 & BD Pharmingen \\
\hline CD62L & BV711 & SK11 & BD Pharmingen \\
\hline CD95 & BV421 & $\mathrm{DX} 2$ & BD Pharmingen \\
\hline CD123 & PE CY7 & $7 G 3$ & BD Pharmingen \\
\hline CD159A & FITC & REA110 & MILTENYI \\
\hline CD195 & $\mathrm{PE}$ & 3A9 & BD Pharmingen \\
\hline CD197 & BUV395 & 150503 & BD Pharmingen \\
\hline CD279 & BV786 & EH12.1 & BD Pharmingen \\
\hline CD336 & $\mathrm{PE}$ & Z231 & BECKMAN COULTER \\
\hline $\begin{array}{l}\text { HLA-DR } \\
\text { Ki-67 }\end{array}$ & $\begin{array}{l}\text { ECD } \\
\text { PERCP CY5.5 }\end{array}$ & $\begin{array}{r}\text { IMMU-357 } \\
\text { B56 } \\
\end{array}$ & $\begin{array}{r}\text { BECKMAN COULTER } \\
\text { BD Pharmingen }\end{array}$ \\
\hline
\end{tabular}

439 


\section{REFERENCES}

4421 Takeda, K., Kaisho, T. \& Akira, S. Toll-like receptors. Annu Rev Immunol 21, 443 335_376, doi: 10.1146/annurev.immunol.21.120601.141126 (2003).

4442 Estes, J. D. et al. Damaged intestinal epithelial integrity linked to microbial 445 translocation in pathogenic simian immunodeficiency virus infections. PLoS

446 Pathog 6, e1001052, doi:10.1371/journal.ppat. 1001052 (2010).

4473 Brenchley, J. M. et al. Microbial translocation is a cause of systemic immune 448 activation in chronic HIV infection. Nat Med 12, 1365_1371, doi: nm1511

$449 \quad$ [pii]

$45010.1038 / \mathrm{nm} 1511$ (2006).

4514 Evans, T. I. et al. SIV-induced Translocation of Bacterial Products in the Liver

$452 \quad$ Mobilizes Myeloid Dendritic and Natural Killer Cells Associated With Liver 453 Damage. J Infect Dis, doi: 10.1093/infdis/jiv404 (2015).

4545 Madsen, K. et al. Probiotic bacteria enhance murine and human intestinal 455 epithelial barrier function. Gastroenterology 121, 580_591,

456 doi:10.1053/gast. 2001.27224(2001).

4576 Marteau, P. R., de Vrese, M., Cellier, C. J. \& Schrezenmeir, J. Protection from 458 gastrointestinal diseases with the use of probiotics. Am J Clin Nutr 73, 430S $459 \quad 4365(2001)$.

4607 Looijer_van Langen, M. A. \& Dieleman, L. A. Prebiotics in chronic intestinal 461 inflammation. Inflamm Bowel Dis 15, 454_462, doi: 10.1002 / ibd. 20737 $462 \quad$ (2009).

4638 Shen, J., Zuo, Z. X. \& Mao, A. P. Effect of probiotics on inducing remission and 464 maintaining therapy in ulcerative colitis, Crohns disease, and pouchitis: meta-analysis of randomized controlled trials. Inflamm Bowel Dis 20, 21_35, doi: 10.1097 / 01. MIB. 0000437495.30052. be (2014).

9 Saez_Lara, M. J., Gomez_Llorente, C., Plaza_Diaz, J. \& Gil, A. The role of probiotic lactic acid bacteria and bifidobacteria in the prevention and treatment of inflammatory bowel disease and other related diseases: a systematic review of randomized human clinical trials. BioMed research international 2015, 505878, doi: 10.1155/2015/505878 (2015). Rioux, K. P. \& Fedorak, R. N. Probiotics in the treatment of inflammatory

47411 Sartor, R. B. Therapeutic manipulation of the enteric microflora in 475 inflammatory bowel diseases: antibiotics, probiotics, and prebiotics. 
Gastroenterology 126, 1620_1633, doi: 10.1053/j.gastro. 2004.03.024 (2004).

12 de Kivit, S., Tobin, M. C., Forsyth, C. B., Keshavarzian, A. \& Landay, A. L. Regulation of Intestinal Immune Responses through TLR Activation: Implications for Pro- and Prebiotics. Front Immunol 5, 60, doi:10.3389 / fimmu. 2014.00060 (2014).

13 Zhong, Y., Huang, J., Tang, W., Chen, B. \& Cai, W. Effects of probiotics, probiotic DNA and the CpG oligodeoxynucleotides on ovalbumin-sensitized

14 Kant, R., de Vos, W. M., Palva, A. \& Satokari, R. Immunostimulatory CpG Brown_Norway rats via TLR9 / NF_kappaB pathway. FEMS Immunol Med

16 Irvine, S. L., Hummelen, R. \& Hekmat, S. Probiotic yogurt consumption may motifs in the genomes of gut bacteria and their role in human health and disease. J Med Microbiol 63, 293_308, doi: 10.1099/jmm.0.064220_0 (2014).

15 Hummelen, R. et al. Effect of 25 weeks probiotic supplementation on immune function of HIV patients. Gut Microbes 2, 80_85, doi: 10.4161/gmic. 2.2.15787 (2014).

$501 \quad 18$ Villar_Garcia, J. et al. Effect of probiotics (Saccharomyces boulardii) on 502 microbial translocation and inflammation in HIV_treated patients: a double_blind, randomized, placebo_controlled trial. J Acquir Immune Defic Syndr 68, 256-263, doi: 10.1097/QAI.0000000000000468 (2015).

19 Ortiz, A. M. et al. IL-21 and probiotic therapy improve Th17 frequencies, microbial translocation, and microbiome in ARV-treated, SIV-infected macaques. Mucosal immunology, doi:10.1038/mi.2015.75 (2015).

20 Klatt, N. R. et al. Probiotic/prebiotic supplementation of antiretrovirals improves gastrointestinal immunity in SIV_infected macaques. J Clin Invest 123,903_907, doi: 10.1172/JCI66227 (2013). 
51121 Komiyama, K., Hirsch, H. Z., Moro, I., Umemura, S. \& Mestecky, J. HNK_1+

512 ( Leu-7) cells and natural killer cell activity in inflamed human gingival tissue.

$513 \quad$ J Oral Pathol 17, 118-123 (1988).

51422 Stelin, S., Ramakrishan, H., Talwar, A., Arun, K. V. \& Kumar, T. S. Immunohistological analysis of CD1a langerhans cells and CD57 natural killer cells in healthy and diseased human gingival tissue: A comparative study. $J$ Indian Soc Periodontol 13, 150_154, doi: 10.4103/0972_124X. 60228 (2009). where commensalism, opportunistic behavior and frank pathogenicity lose their borders. AIDS 26, 1457-1472, doi: 10. 1097/QAD. 0b013e3283536ba8 (2012).

Gregory, C. D., Murray, R. J., Edwards, C. F. \& Rickinson, A. B. Downregulation of cell adhesion molecules LFA_3 and ICAM_1 in EpsteinBarr virus positive Burkitts lymphoma underlies tumor cell escape from virus_specific T cell surveillance. J. Exp. Med. 167, 1811_1824 (1988).

53628 Spits, H. , Bernink, J. H. \& Lanier, L. NK cells and type 1 innate lymphoid cells:

29 Spits, H. et al. Innate lymphoid cells_--a proposal for uniform nomenclature. Nat Rev Immunol 13, 145_149, doi: 10.1038/nri3365 (2013). we miss them? Nat Rev Immunol 13, 75_87, doi:10.1038/nri3349 (2013).

31 Klose, C. S. \& Artis, D. Innate lymphoid cells as regulators of immunity, inflammation and tissue homeostasis. Nat Immunol 17, 765_774, doi: $10.1038 /$ ni. 3489 (2016). 
54632 Shah, S. V., Manickam, C., Ram, D. R. \& Reeves, R. K. Innate Lymphoid Cells in HIV / SIV Infections. Frontiers in immunology 8, 1818, doi:10.3389/fimmu. 2017.01818 (2017).

$54933 \mathrm{Xu}, \mathrm{H}$. et al. IL_17-producing innate lymphoid cells are restricted to mucosal $550 \quad$ tissues and are depleted in SIV_infected macaques. Mucosal Immunol 5, 658_ 669, doi: 10.1038/mi.2012.39 (2012).

$55234 \quad$ Reeves, R. K. et al. Gut inflammation and indoleamine deoxygenase inhibit IL-17 production and promote cytotoxic potential in NKp44+ mucosal NK cells during SIV infection. Blood 118, 3321-3330, doi: 10.1182/blood-2011_ 04-347260 (2011).

35 Mudd, J. C. et al. Hallmarks of primate lentiviral immunodeficiency infection recapitulate loss of innate lymphoid cells. Nat Commun 9, 3967, doi:10.1038/s41467_018_05528_3 (2018). during Acute HIV-1 Infection in the Absence of Viral Suppression. Immunity in Beneficial Modulation of Mucosal and Systemic Immune Function. $J$ Immunol 196, 2401_2409, doi:10.4049/jimmunol.1502470 (2016). Hamza, O. J. et al. Oral manifestations of HIV infection in children and adults receiving highly active anti-retroviral therapy [HAART ] in Dar es Salaam, Tanzania. BMC Oral Health 6, 12, doi:10.1186/1472_6831_6-12 (2006). Noguera-Julian, M. et al. Oral microbiome in HIV_associated periodontitis. Medicine (Baltimore) 96, e5821, doi: 10.1097/MD. 0000000000005821 (2017).

Ortiz, A. M. et al. IL_21 and probiotic therapy improve Th17 frequencies, microbial translocation, and microbiome in ARV_treated, SIV-infected macaques. Mucosal Immunol 9, 458_467, doi:10.1038/mi. 2015.75 ( 2016 ).

41 Klatt, N. R. et al. Probiotic/prebiotic supplementation of antiretrovirals improves gastrointestinal immunity in SIV_infected macaques. J Clin Invest, doi: 10.1172/JCI66227 (2013).

42 Barouch, D. H. et al. Vaccine protection against acquisition of neutralizationresistant SIV challenges in rhesus monkeys. Nature 482, 89_93, doi: 10.1038 / nature10766 (2012). 
58043 Mattapallil, J. J. et al. Massive infection and loss of memory CD4+ T cells in multiple tissues during acute SIV infection. Nature 434, 1093_1097, doi: 10.1038 / nature03501 (2005).

44 Veazey, R. S. et al. The gastrointestinal tract as a major site of CD4 T lymphocyte depletion and viral replication in SIV infection. Science 280, 427

45 Li, H. et al. Hypercytotoxicity and Rapid Loss of NKp44+ Innate Lymphoid

46 Ishizaki, A. et al. Effects of Short-Term Probiotic Ingestion on Immune

47 Villar_Garcia, J. et al. Impact of probiotic Saccharomyces boulardii on the gut microbiome composition in HIV_treated patients: A double_blind,

48 Whitney, J. B. et al. Rapid seeding of the viral reservoir prior to SIV viraemia

49 Caporaso, J. G. et al. Global patterns of 16 S rRNA diversity at a depth of millions of sequences per sample. Proc Natl Acad Sci U S A 108 Suppl 1, 4516_4522, doi: 10.1073/pnas. 1000080107 (2011).

602

603 randomised, placebo_controlled trial. PLoS One 12, e0173802,

604 